\title{
A HIGH-RATE, HIGH-RESOLUTION ASYMMETRIC WIRE CHAMBER WITH MICROSTRIP READOUT
}

\author{
G. Charpak, I. Crotty, Y. Giomataris, L. Ropelewski \\ and M.C.S. Williams \\ CERN, Geneva, Switzerland
}

\begin{abstract}
We have investigated the properties of an asymmetric wire chamber with cathode strip readout. The use of a small gap between the anode plane and the cathode plane and of an electric field configuration provide fast removal of the positive ions produced in the avalanche process and restrict the area of the induced signal on strips engraved on the cathode plane. We can thus contemplate using digital readout for these strips and still have good position resolution, or use analog readout for high spatial resolution. The detector can operate at very high gas gain $\left(10^{5}\right)$; thus with a small gap of $2 \mathrm{~mm}$ we can achieve full efficiency for minimum ionizing particles. We have measured no gain modification at a rate of $2 \times 10^{5}$ particles $\mathrm{mm}^{-2} \mathrm{~s}^{-1}$ at a gain of $10^{4}$ with a distance of $600 \mu \mathrm{m}$ between anodes and cathodes and $500 \mu \mathrm{m}$ between anode and cathode field wires.
\end{abstract}

Submitted to Nuclear Instruments and Methods in Physics Research, A 
The use of high-granularity position-sensitive detectors is a challenge for charged particle tracking in future high-luminosity particle accelerators. Many applications of such detectors are also under discussion in nuclear medicine, biology, and other domains. The maximum rate capability of these devices and their ageing resistance in strong irradiation environments is a fundamental issue. This demand is partially covered by the development of silicon microstrip detectors and recent progress in microstrip gaseous chambers (MSGCs) [1]-[4]. The MSGCs have attracted considerable interest for use at future high luminosity colliders owing to their high rate and high accuracy capabilities. Their application is, however, limited to small surface coverage.

The asymmetric chamber consists of an anode wire plane mounted close to the cathode plane with engraved pick-up strips running orthogonal to the anode wire direction. This allows a very short path for the positive ions created in the avalanche, thus reducing the collection time of the positive ions and increasing the rate capability of the device by reducing the space-charge effect. A larger gap on the other side of the anode wire plane allows a sufficient number of primary electron-ion pairs to be generated.

Asymmetric wire chambers have been considered since the beginning of the development of wire chambers [5]. Many groups have investigated this structure for various applications [6]. For instance, in order to increase the size of signals induced on pads, a gap as small as $0.4 \mathrm{~mm}$ has been used for a photodetector [7].

Our main objective is to optimize the rate capabilities of these types of chambers. We are also interested in the position resolution of the microstrip readout. The position can be derived by two means, the first being with analog techniques, where the charge on each strip is measured and the position given by the centre of gravity or some similar algorithm [8]-[10]. This is possible at low-rate experiments (such as LEP experiments) as many channels can be multiplexed together to a few analog-to-digital converter (ADC) channels. This method is limited at high-luminosity colliders since the channels cannot be multiplexed owing to the high rate. Thus we are also interested in digital readout. If we can identify the strip with the biggest charge we can get a resolution limited by the strip width (i.e. $\sigma=144 \mu \mathrm{m}$ for our $500 \mu \mathrm{m}$ width strips). This can be further improved if we can identify the case when two strips have comparable charges; thus even with digital readout we can foresee a resolution better than $100 \mu \mathrm{m}$. In order to design optimal electronics it is necessary to investigate the charge sharing between both strips.

\section{Description of the chamber}

Figure 1 shows the cross-section of the chamber. The anode plane consists of alternating thin $7 \mu \mathrm{m}$ diameter anode wires and $20 \mu \mathrm{m}$ field wires on a $500 \mu \mathrm{m}$ pitch. The plane of pick-up strips is located $600 \mu \mathrm{m}$ below, and has strips on a $400 \mu \mathrm{m}$ pitch orthogonal to the anode wires. Cathode strips are printed on a ceramic plate of a thickness that could, in principle, be reduced to a few hundred microns. Strips are connected to the ground through the preamplifier. There is a gas gap of $3 \mathrm{~mm}$ on the other side, closed by a metallic mesh foil. This plane is connected to a negative voltage, which is set at a typical value of $1500 \mathrm{~V}$. Both the anode wires and the cathode strips are read out with standard preamplifiers having $300 \mathrm{mV} / \mathrm{pC}$ sensitivity. The gas filling was $80 \% \mathrm{Ar}$ and $20 \% \mathrm{C}_{2} \mathrm{H}_{6}$.

\section{$3 \quad$ Results and measurements}

A typical signal from the anode obtained with a minimum ionizing particle normal to the anode plane is shown in Fig. 2. Similar signal shape was obtained from the cathode 
strips with reverse polarity. The ratio between the collected charge on the neighbouring strip to that collected on the wire was measured to be $40 \%$. The rise time of the signal is 15 ns including some contribution of the preamplifier time constant. Clearly the response of the detector is satisfactory; with a more sophisticated current preamplifier and a suitable shaping it can be faster. The gain obtained as a function of the high voltage applied on the wires is shown in Fig. 3. At the value of $1000 \mathrm{~V}$ on the anode wires, with $50 \mathrm{~V}$ on the field shaping wires the useful gain was measured to be $10^{5}$. At that gain the detector is able to detect single electrons; therefore it needs only a modest number of primary electrons produced per minimum ionizing particle and the sensitive gap can be reduced. A narrow gap is an important advantage, since the position resolution of the detector for inclined tracks is improved.

The behaviour of the chamber was tested with an ${ }^{55} \mathrm{Fe}$ radioactive source. The pulseheight distribution obtained is shown in Fig. 4. The expected peak at $5.9 \mathrm{keV}$ is seen to be well separated from the Ar escape peak; it is rather uniform over the sensitive area of the detector with $\sigma=6 \%$. This is a satisfactory result for most applications and it can be improved with a careful choice of the appropriate gas and electronic chain.

To check the rate capability of the device, the chamber was exposed to strong radiation by an X-ray gun with a variable flux of X-rays at the energy of $8 \mathrm{keV}$. The gas filling in this case was Ar 90\% and DME (dimethylether) 10\%. The X-ray flux and the total current seen by the anode wires were monitored during measurements. The test was performed at the gas gain of $10^{4}$. Figure 5 shows the normalized gain as a function of the number of particles per $\mathrm{mm}^{2}$ hitting the detector. The number of X-rays irradiating the detector was measured at the same time as the gain and the total current seen by the anode wires. The measurement was performed at a gain of $10^{4}$. The gain remains stable up to a particle rate of $2 \times 10^{5} \mathrm{~mm}^{-2} \mathrm{~s}^{-1}$ and there is a loss of only $30 \%$ at $10^{6} \mathrm{~mm}^{-2}$ $\mathrm{s}^{-1}$. The maximum rate of operation of this device is more than one order of magnitude higher than that obtained with conventional proportional chambers. It reflects the effect of the fast evacuation of the positive ions through the small gap. This effect is illustrated in Fig. 6, which is a plot of the electric field lines around the anode wires. The major part of the positive ion charge $(\approx 80 \%)$, created closed to the anode wires, is collected by the field wires or cathode strips.

\section{Conclusions and outlook}

The microstrip asymmetric gaseous detector has been shown to be a promising device for use in large-area tracking devices in very-high-radiation environments. It operates up to $10^{6} \mathrm{~mm}^{-2} \mathrm{~s}^{-1}$. Our tests have shown that a significant increase in rate capability can be obtained by a wire structure that permits a more rapid evacuation of the positive ions from the avalanches. The rate capability which we have observed, $3 \times 10^{5} \mathrm{~mm}^{-2} \mathrm{~s}^{-1}$, has to be compared with tests giving a few $10^{6}$ obtained with MSGCs, but at a gain of only $10^{3}$. Thus the rate capabilities are comparable. A similar work was done by a group at Siegen [11]. The structure of our chamber allows stereo tracking if anode wires are read out. Although the accuracy is limited by the wire spacing it can give valuable information, especially to remove ambiguity. In addition, the time resolution is significantly better than the MSGC. With the parameter chosen in the present design the rise time is $15 \mathrm{~ns}$ and the time resolution is expected to be of the order of a few nanoseconds. It is thus well adapted to the need of the future high-luminosity proton collider experiments.

The size of the detector can be large. Using narrow spacers, made for instance of insulating wires of a few hundred microns every $5 \mathrm{~cm}$, the distance between anode wires 
and the cathode strip plane can be kept very constant, without significant efficiency loss. The chamber also allows a certain flexibility in the readout system, for instance microstrips placed on the back of a semiconducting cathode can be used.

A schematic of an improved microstrip asymmetric chamber is illustrated in Fig. 7. The small cathode-anode gap is reduced to $200 \mu \mathrm{m}$ by using insulating spacers, whilst the large ionization gap is $2 \mathrm{~mm}$. The strip size is also rescaled to $200 \mu \mathrm{m}$. Furthermore, the anode spacing has to be reduced to increase the performances of the chamber, which is technically hard to achieve with the present design. It can, however, be accomplished by putting the field wires in a second plane, at a distance of $200 \mu \mathrm{m}$ above the anode plane. This new configuration also allows faster positive-ion charge evacuation through a smaller gap and reduces that part of the ions drifting in the larger gap. The simulation program shows that almost $99 \%$ of the space charge is diffusing and is collected on the field wires or the cathode strips. A rough estimate of the ion drift time through the small gap $(100 \mu \mathrm{m})$ shows that it can be of the order of $100 \mathrm{~ns}$ for an optimized gas mixture. It will permit an improvement of the output signal, a very-high rate capability of this detector, and a fine granularity giving a precise stereo spatial point of the incident charged particle.

\section{Acknowledgements}

We would like to thank R. Bouclier for having built the first prototype and M. Price for the preparation of the wire frame.

\section{References}

[1] A. Oed et al., Nucl. Instrum. Methods A263 (1988) 351.

[2] F. Angelini et al., Nucl. Instrum. Methods A283 (1989) 755.

[3] M.H.J. Gijberts et al., Nucl. Instrum. Methods A313 (1992) 377.

[4] R. Bouclier et al., Nucl. Instrum. Methods A323 (1992) 236.

[5] G. Charpak, D. Rahm and H. Steiner, Nucl. Instrum. Methods 80 (1970) 13.

[6] M. Atac, Nucl. Instrum. Methods 176 (1980) 1.

[7] R. Arnold et al., Nucl. Instrum. Methods A314 (1992) 465.

[8] G. Charpak et al., Nucl. Instrum. Methods 148 (1978) 471.

[9] A.H. Walenta, Nucl. Instrum. Methods 151 (1978) 462.

[10] V. Radeka and R.B. Boie, Nucl. Instrum. Methods 178 (1980) 543.

[11] E. Roderburg et al., Nucl. Instrum. Methods A323 (1992) 140. 


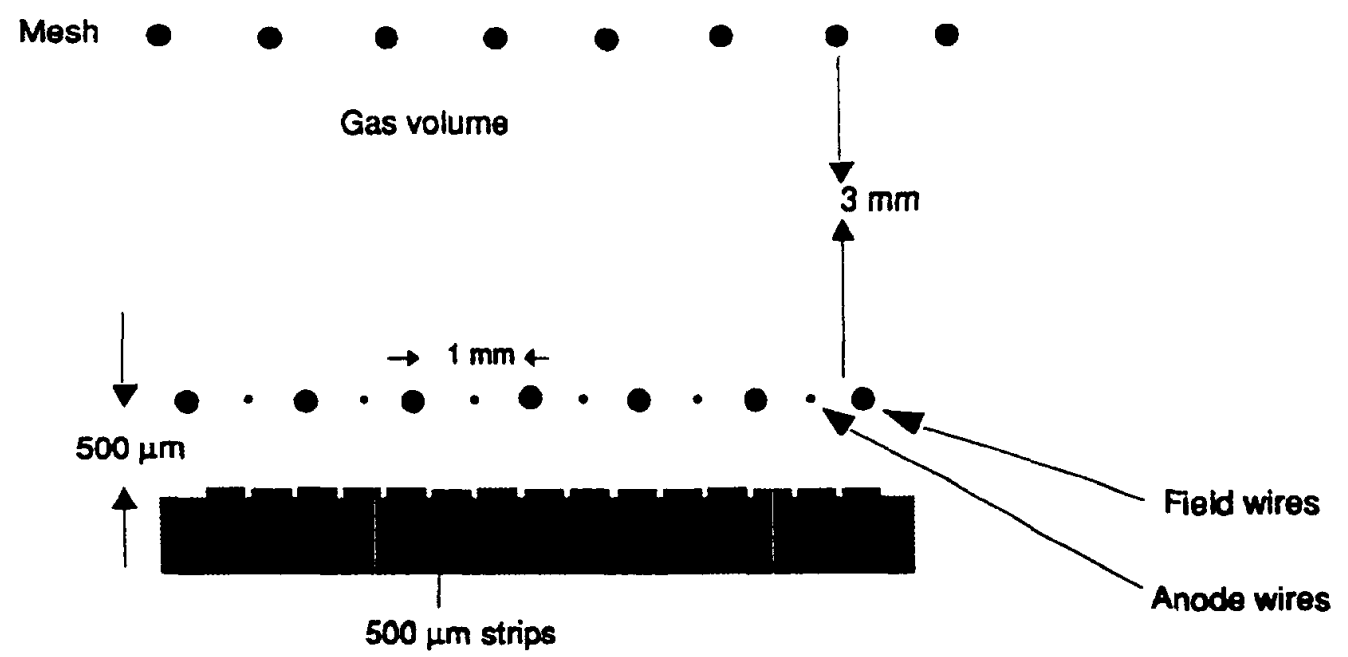

Figure 1. Schematic of the cross-section of the asymmetric wire chamber.

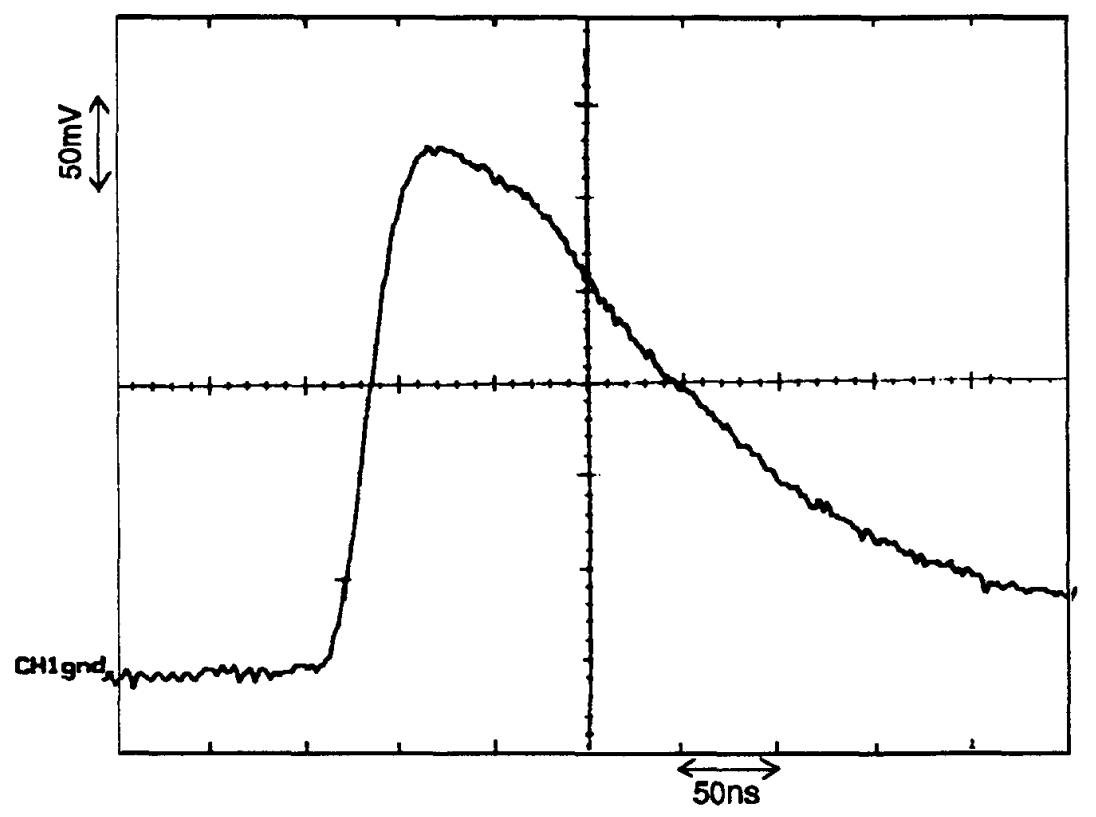

Figure 2. A typical pulse after amplification observed with a digital oscilloscope. The pulse amplitude (50 $\mathrm{mV}$ per division) is plotted against time (50 ns per division). 


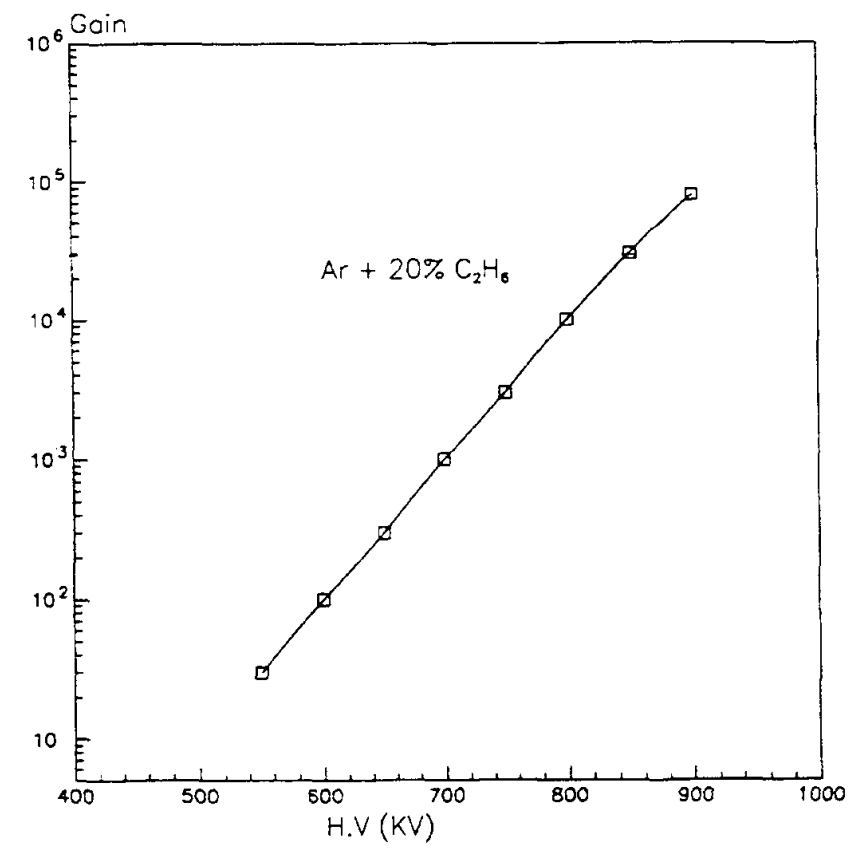

Figure 3. Useful gain observed as a function of the high voltage applied on the anode wires. For this measurement the voltage applied on the mesh was $1600 \mathrm{~V}$ and on the field wires it was $100 \mathrm{~V}$.

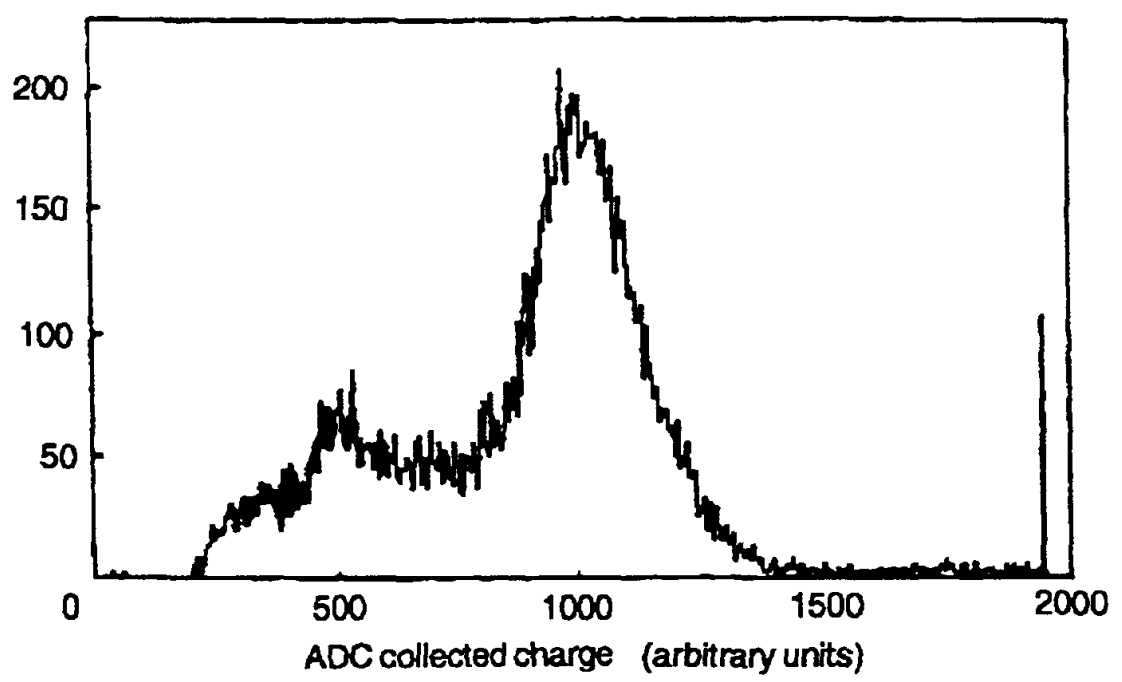

Figure 4. Pulse-height distribution obtained for $5.9 \mathrm{keV}{ }^{55} \mathrm{Fe} \mathrm{X}$-rays (large peak) and the $\mathrm{Ar}$ escape peak (small peak). 


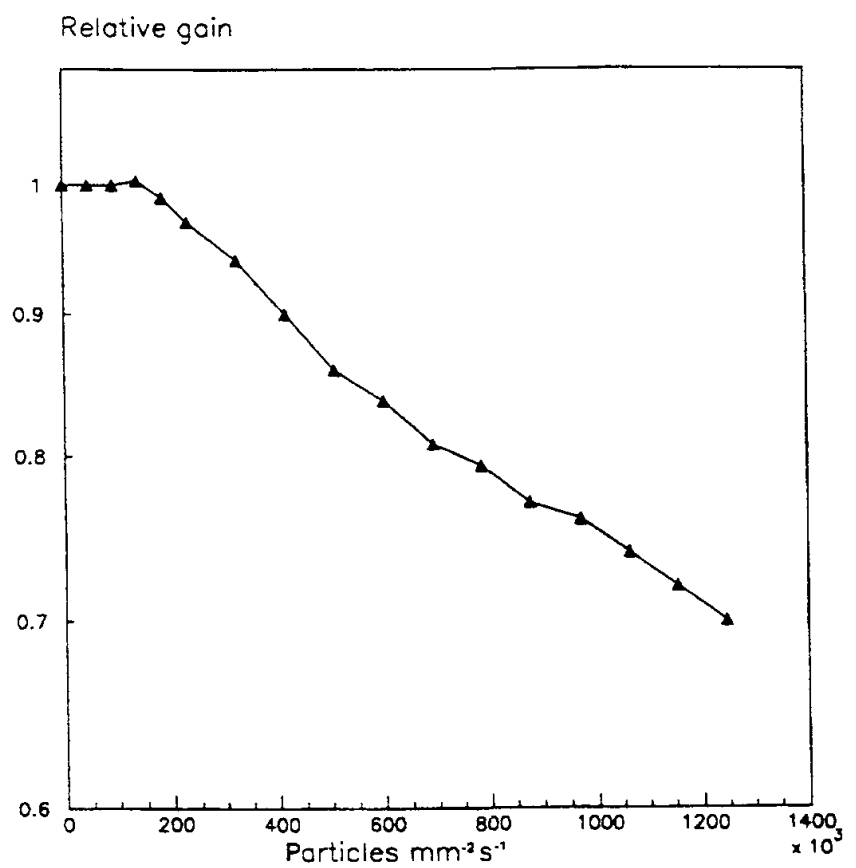

Figure 5. The relative gain (with respect to the maximum gain) as a function of the flux of $\mathrm{X}$-rays converted inside the detector. The measurement was performed at a gain of the chamber of $10^{4}$.

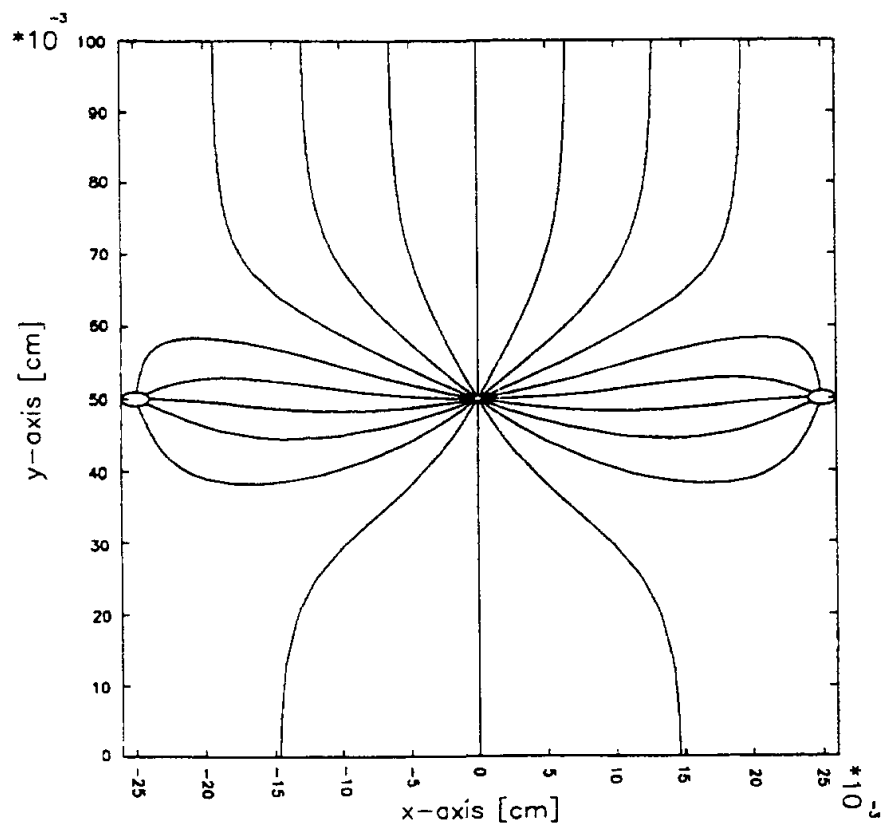

Figure 6. Simulation of the field lines in a drift cell. 


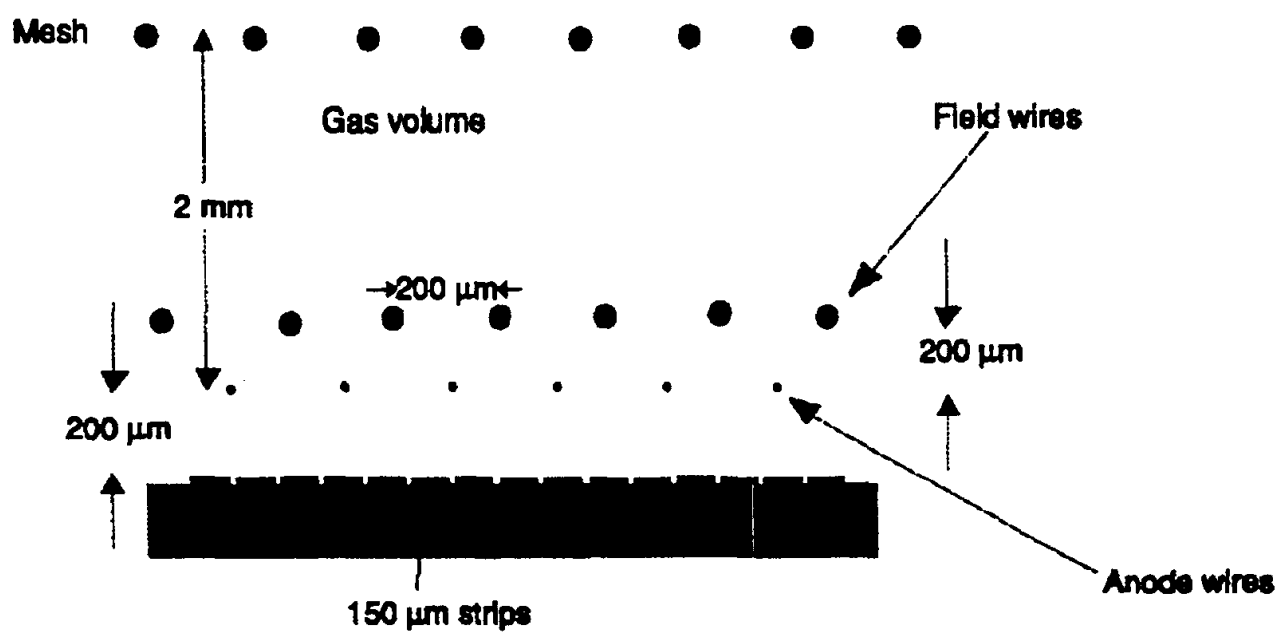

Figure 7. Schematic of the cross-section of an optimized asymmetric wire chamber. The chamber design parameters are extended in order to achieve higher performance. 
